\title{
Mediación y medio ambiente
}

\author{
Mediation and environment
}

José Antonio Serrano Morán

Universidad Autónoma de Nayarit, México serranomoran@gmail.com

Francisco Javier Rivas Sandoval Universidad Autónoma de Nayarit, México rivas115@hotmail.com

Humberto Lomelí Payán

Universidad Autónoma de Nayarit, México lomeliuan@hotmail.com

Miguel Ángel Anaya Ríos

Universidad Autónoma de Nayarit, México miguelanaya2000@hotmail.com

\section{Resumen}

El presente estudio tiene como objetivo fundamental el desentrañar la viabilidad y funcionabilidad de la utilización del mecanismo de mediación para resolver conflictos de carácter ambiental, haciendo alusión a las legislaciones que deben entrar a la regulación de tal escenario y los participantes directos en la aplicabilidad de este mecanismo alterno.

Para tales fines, se tomó como base el método deductivo, al partir de una ideología superior que se cimienta en la justicia restaurativa y la autocomposición de manera general, hasta arribar de manera específica a la resolución de conflictos en materia ambiental, a través de mecanismos no jurisdiccionales, de igual forma fueron utilizados los métodos, documental, exegético, sistemático jurídico y dialéctico.

Situación que nos arroja como resultado, la identificación del debido uso de la herramienta de mediación en asuntos de carácter ambiental, su debida aplicación y funcionabilidad. 
Palabras Clave: mediación, medio ambiente, conflicto social.

\section{Abstract}

The present study has as main objective to unravel the viability and functionality of the use of mediation mechanism to resolve disputes environmental, referring to the laws that must enter the regulation of such a scenario and the direct participants in the applicability of this alternative mechanism.

To that end, it took as a basis the deductive method, from a superior ideology is founded on restorative justice and autocomposición generally, until arriving specifically to conflict resolution in environmental matters, through mechanisms non-judicial, just as the methods were used, documentary, exegetical, systematic legal and dialectical, as well.

This situation casts us as a result, identification of the proper use of the tool of mediation in matters of environmental, its proper application and functionality.

Key Words: mediation, environment, social conflicto.

Fecha Recepción: Agosto 2015 Fecha Aceptación: Enero 2016

\section{INTRODUCCIÓN}

Hablar sobre conflictos ambientales es tratar un tema que no se limita a solo una parte de la población o un puñado de personas, es tratar un tema que impacta en todos los niveles y estratos sociales, de ahí su relevancia y especial análisis, es por eso que en el presente artículo se pretende identificar de manera clara las razones que dan origen a los conflictos ambientales que con posterioridad repercuten como problemas ambientales, a efecto de dotarlos de una solución de fondo, sin embargo, le hemos dado un enfoque distinto al incorporar la justicia alternativa, más en específico a los mecanismos alternativos de solución de controversias y específicamente a su herramienta de la mediación, como resolutor final de los conflictos ambientales.

Sin duda un mecanismo alterno de solución de conflictos que se basa en el dialogo, la empatía, la tolerancia y el rapport, para generar cambios culturales en las personas participantes, situación que se aleja de la ideología de que los conflictos solo se pueden resolver a través de más 
conflictos, es decir, a través de litigios en organismos de impartición de justicia formal, los cuales se han convertido en procesos estigmatizantes, burocráticos, oscuros y corruptos, y que no proporcionan una solución equitativa o inclusive justa para ninguna de las partes, lo cual no garantiza la finalidad ulterior para lo cual fue diseñado y estructurado el sistema de impartición de justicia, nos referimos a la no repetición del acto.

Es por lo ya señalado, que consideramos que el uso de la mediación en la resolución de conflictos ambientales, es una herramienta útil, funcional, pragmática y confiable para ejecutar la tarea que la sociedad la ha conferido al Estado, la protección de su integridad y el garantizar la perpetuación de la especie, lo anterior, al atacar el fondo del asunto y no solo sus consecuencias, teniendo en mente que este mecanismo por si solo no contara con los resultados anhelados, sino que debe de venir acompañado de políticas públicas encaminadas hacia el mismo objetivo, hacia la construcción de una sociedad eco-amigable y ambientalmente responsable.

\section{JUSTICIA RESTAURATIVA COMO IDEOLOGÍA DE RESOLUCIÓN DE CONFLICTOS}

Actualmente, se ha venido revolucionando la forma en que comprendemos, percibimos y combatimos el delito, cada día más nos encontramos con ideas y filosofías que nos llevan de la mano hacia un sistema de mínima intervención del Estado para solución los conflictos que emanan entre los individuos.

Uno de estos nuevos paradigmas de combate al conflicto, y tal vez uno de los que está causando mayor revuelo por sus contundentes resultados en el ámbito tanto nacional como internacional, es el de la Justicia Restaurativa, fenómeno jurídico que nos propone una nueva visión frente al ilícito, en donde lo más importante no es ya el de castigar al presunto responsable, sino el de atender las causas últimas que lo llevaron desarrollar su conducta, y sobre todo darle la oportunidad de contribuir en resolver el tejido social que ha dañó, a través de herramientas de dialogo y pacificación.

De igual forma, la Justicia Restaurativa viene a otorgar a la víctima, el papel protagonista dentro de la resolución de un conflicto, al poner a su disposición una serie de herramientas, basadas en el dialogo, comprensión, tolerancia y empatía por los demás, que podrá utilizar en pro de sus pretensiones, siendo la mediación, el instrumento por excelencia de la Justicia Restaurativa. 
Ahora bien, para un mayor entendimiento de esta nueva filosofía de impartición y administración de justicia, analizaremos diversas definiciones que se han venido construyendo a lo largo de los años, con la finalidad de arribar a un concepto propio que nos pueda satisfacer de manera completa.

El maestro Tony Marshall definió a la Justicia Restaurativa como "un proceso por el cual las partes con un interés específico en un delito, colectivamente resuelven cómo hacer frente a las consecuencias de la infracción y sus implicaciones para la futuro" (Marshall, 1999)

De la anterior definición podemos concluir que Marshall le da demasiada importancia al proceso y las partes involucradas en el mismo, dejando de lado tal vez lo más relevante, que es la restauración y reparación del tejido dañado por el conflicto, situación por la cual surgieron nuevos tratadistas que intentaron enmendar esta situación, de los cuales podemos mencionar a Gordon Bazemore y Lode Walgrave, quienes en su definición manifiesta que se trata de "toda acción orientada principalmente a hacer justicia a través de la restauración o reparación del daño causado por el delito" (Bazemore \& Walgrave, 1999)

Enunciado del cual se desprende un nuevo elemento, el de la justicia, elemento que viene a ser el génesis de todo este nuevo paradigma, ya que es la idea del arribo a una justicia verdadera lo que nos impulsa a investigar, reinventar y redescubrir todas y cada una de las figuras jurídicas que utilizamos día a día, y en el caso particular, el de la Justicia Restaurativa.

Sin embargo, si un elemento torna único al fenómeno de la Justicia Restaurativa, es el hecho de que involucra para la resolución de los delitos, no solo a las partes actoras en el mismo, sino a las personas o comunidades que se vieron afectadas por los hechos delictivos, permeando a mayor escalas los efectos benéficos que acarrea esta nueva visión de combate al delito, ya que nos permitirá sanar y reconstruir el tejido social y las relaciones interpersonales que se han visto dañadas y que no pueden ser subsanadas simplemente con la garantiza de la reparación del daño, de igual forma, le permitirá a la comunidad, recobrar la confianza en el estado, como órgano protector y defensor de su integridad y derechos básicos.

Por todo lo anterior podemos definir a la Justicia Restaurativa como el resultado que arrojarán los diversos mecanismos informales en los cuales intervienen los actores y los miembros de la sociedad que fueron afectados por el conflicto, a efecto de reparar y resarcir los daños ocasionados a consecuencia del antijurídico, tomando en consideración todas y cada una de las pretensiones externadas por los partícipes en el proceso de restauración. 
Este paradigma o filosofía de seguridad pública cuenta con diversos principios y características que lo dotan de funcionabilidad, situación que a continuación analizaremos.

Algunos autores como Daniel W. Van Ness y Karen Heeterks Strong, señalan que para poder estar en presencia de una justicia restaurativa tenderemos que tomar en cuenta cuatro valores, el primero, el encuentro entre las partes y su comunidad, en donde se les permite mostrar emociones para facilitar el entendimiento mutuo, el segundo, es la reparación del daño causado a la víctima y después a la sociedad, el tercero, es la reintegración, la cual se refiere tanto a la víctima como al autor del delito, persiguiendo que se inserte nuevamente como persona íntegra en la comunidad, y por último, está la participación dada por la oportunidad que se otorga a las partes involucradas activamente en todas las etapas del proceso. (Vannes \& Strong, 2001)

De igual forma, algunos otros actores como Susan Sharpe (Kemelmajer) y Eric Caballero Sepúlveda, (Caballero Sepulveda, 2009) coinciden en algunas características claves, tales como la voluntariedad, la reparación del daño, la reparación del tejido social y la participación directa de los sujetos activos del ilícito en conjunto con la sociedad, sin embargo, podemos enlistar las características y principios sin las cuales son podríamos hablar de una justicia restaurativa.

Siendo estas, a) La participación de todos los afectados, en donde la participación directa en la resolución del conflicto, de los protagonistas del conflicto en conjunto con miembros de la sociedad que también se vieron afectados por el mismo, en busca de una solución holística y de medidas que eviten la repetición de los hechos.

b) Se le da prioridad a las medidas encaminadas a la restitución y no la prisión, toda vez que La finalidad ulterior debe ser la reparación de las relaciones interpersonales y el tejido social, sin que esto signifique la extinción de la sanción para el responsable, c) La voluntariedad, elemento clave en los procesos de justicia restaurativa, toda vez que sin elemento volitivo de las partes, difícilmente se daría un resultado reparador o transformativo, ya que no podríamos concientizar al ofensor del daño que ha causado, y mucho menos logar su arrepentimiento por sus hechos, d) El reconocimiento del daño ocasionado, el ofensor debe ser capaz de visualizar los alcances de su conducta, a efecto de poder dar el primer paso a la reparación del daño ocasionado, a través de verse frente a frente con los sujetos que ha dañado, y poniéndose en el lugar de ellos, imaginando al mismo tiempo que sentiría si el afectado fuera alguien cercano o bien si fuera él mismo, y e) La reconstrucción del tejido social, esta es la característica más importantes de la justicia restaurativa, ya que se trata de la reparación de las relaciones entre víctima, victimario y 
sociedad, pero sociedad no como un ente abstracto, sino como el cúmulo de personas que también se vieron afectada por el hecho.

\section{LA MEDIACIÓN COMO MECANISMO PACIFICADOR}

El mecanismo de la mediación es un tema ahora en nuestros días que cuenta con más estudio, que se encuentra más desarrollado y que poco a poco ha permeado en nuestra sociedad, es así que podemos encontrar un buen número de definiciones sobre la mediación, de las cuales cobran relevancia las siguientes, "La mediación es un método alternativo no adversarial para logar la solución de conflictos, mediante el cual uno o más mediadores, quienes no tienen facultad de decisión, intervienen únicamente facilitando la comunicación entre los interesados en el conflicto, con el propósito de que ellos acuerden voluntariamente una solución que ponga fin al mismo, total o parcialmente, y también coadyuvar para lograr una paz social.” (Hidalgo Murillo, 2010)

Como podemos observar, en esta definición se le da prioridad a la participación directa que tienen las partes al momento de resolver el conflicto con la ayuda de un tercero imparcial, que solo se limita a facilitar el dialogo, toda vez que como lo hemos comentado, la solución surge directamente de los protagonistas del propio conflicto y de nadie más.

De igual forma analizamos otras definiciones para el tema que abordamos y las cuales nos brindan un mejor entendimiento, "La mediación constituye un procedimiento de resolución de disputas flexible y no vinculante, en el cual un tercero neutral - el mediador - facilita las negociaciones entre las partes para ayudarlas a llegar a un acuerdo.” (Pastrana Aguirre, 2009)

En ese mismo sentido Pacheco pulido señala que la justicia restaurativa "Es un procedimiento en el cual dos o más personas que tienen un problema en común, solicitan el apoyo de un tercero que facilita la comunicación entre ellos, para que de manera pacífica y equitativa, lleguen a un acuerdo satisfactorio para ambas partes." (Pachecho Pulido, 2012)

Por último Soleto Muñóz y Ortero Parga nos dicen que es "Una forma para la resolución alternativa del conflicto, a través de la cual las partes en conflicto llegan por si mismas a una solución con la ayuda de un mediador, que con su formación ofrece a las partes nuevas vías de diálogo y entendimiento. (Soleto Muñoz \& Ortero Parga, 2007)

Estas definiciones centras su atención en la figura del mediador, el tercero imparcial que viene a facilitar y propiciar el dialogo entre las partes en conflicto, a fin de ayudarlas a resolver el fondo de su asunto, personaje que cuenta con una especial relevancia, al ser el artífice de los acuerdos a 
los que puedan arribar las partes, sin el cual la comunicación no sería posible ni mucho menos la resolución de los conflictos, al menos no de forma pacífica.

Es así que podemos observar que se ha considerado a la mediación como un mecanismos que otorga la posibilidad de resolver el génesis de una conflictiva, y el cual al mismo tiempo genera en las partes una nueva visión de diálogo y entendimiento, dotándolos de herramientas para socializar con el resto de la comunidad, evitando en gran medida la reincidencia en el conflicto, teniendo como limitante el respeto a diversos principios y requisitos constitucionales, tales como la presunción d inocencia, el debido proceso, la tutela judicial efectiva, entre otros. (Cervantes Bravo, 2012)

De igual forma, la figura de la mediación cuenta con diversos principios y características que la dotan de las bondades que ya hemos comentados, sin embargo, hemos considerado que los principios regentes de la mediación, sin los cuales no podríamos concebirla son los siguientes.

La voluntariedad, toda vez que hablar de una mediación forzada u obligatorio, es ir encontrar de la propia naturaleza del instrumento alternativo de solución de conflicto, además de que no podríamos lograr una verdadera mediación transformativa, que es el objetivo de toda mediación, así como tampoco podríamos comprometer a las partes a respetar el acuerdo al que pudieren arribar.

La flexibilidad, elemento relevante al permitir que sean las partes protagonistas del conflicto quien dicten las etapas y momentos procesales de la sesión de mediación, sin extralimitarse ni salirse de una guía general que el propio mediador les aporta, lo cual permite aumentar la probabilidad de alcanzar un acuerdo.

La confidencialidad, toda vez que será la red de seguridad que usaran las partes para poder expresar su verdadero sentir, sus reales pretensiones y necesidades, sin el temor de que sus palabras podrán usarse en su contra en un futuro proceso jurisdiccional formal, y por último el carácter no adversarial, con el cual llegamos a ese cambio de paradigma y mentalidad del cual tanto se ha hablado en el nuevo sistema acusatorio, filosofía de seguridad publica en donde lo más importante no es el castigo a una de las partes y el beneficio para la otra, sino el entendimiento entre estas, el arrepentimiento de un lado y el perdón del otro, trayendo como consecuencia la restauración y curación de la relación interpersonal.

Finalmente atendemos el tema de los conflictos que deben ser susceptibles de pasar por un proceso de mediación, en este sentido Zaera Navarrete, nos comenta que no debe de ser usada la 
mediación en conflicto que involucren violencia de género o bien cuando una de las partes presente psicopatología importante, toda vez que en más que ayudar a las partes a resolver su conflicto, estaríamos victimizándolas aún más. (Zaera Navarrete, 2013)

De igual forma, la Dra. Guadalupe Márquez Algara, señala que la mayoría de los conflictos son mediables, al señalar como ejemplo las siguientes áreas, los problemas que se presente dentro de la familia, las relaciones laborales, el medio ambiente, el consumidor, los contratos, la responsabilidades profesionales, los daños y perjuicios, las patentes, las marcas y propiedad intelectual, la propiedad horizontal, la división de condominio, las obligaciones de pago, problemas vecinales, conflictos comunitarios, sucesiones, otros. (Márquez Algara, 2004)

Por lo antes señalado, consideramos que los conflictos que deben de ser susceptibles de pasar por un proceso de mediación aquellos en los que podamos identificar a los protagonistas del mismo, es decir, aquellos en donde podamos identificar los afectados y su relación con la problemática, dando así la oportunidad de ayudar al mayor número de personas que sea posible, evitándoles pasar por un proceso jurisdiccional formal.

\section{LAS CONCEPCIONES Y ORÍGENES DE LOS CONFLICTOS AMBIENTALES}

Actualmente hemos adoptado una cultura de autodestrucción, en donde ponderamos los intereses económicos y placeres mundanos por la protección y el cuidado del medio ambiente, lamentablemente esta cultura que cada vez permea más en la sociedad, y la cual está dejando ver sus grandes consecuencias, tales como inundaciones, sequias, heladas, fenómenos naturales cada más frecuentes y más peligrosos.

Esto no es sino las consecuencias de nuestros actos y el ataque frontal que hemos desencadenado, ya sea de manera consciente o inconsciente hacia el medio ambiente, teniendo como resultado, afectaciones al medio ambiente tan severas como pérdida de biodiversidad, cambio climático, sobrepoblación, contaminación en todas sus modalidades, deforestación entre muchas más, teniendo como ejemplo la contaminación y la constante reducción del agua dulce de nuestro planeta, la cual está destinada a convertirse la preocupación mundial del siglo XXI. (Ávila Verdín, Rosas- Topete, \& Arciniega-Ponce, 2014) 
Sin embargo, antes de entrar al estudio del tema, debemos diferenciar de manera precisa lo que se considera como problema ambiental y lo considerado como conflicto ambiental y conflicto socioambiental.

Un problema ambiental es cualquier alteración que provoca desequilibrio en un ambiente dado, afectándolo negativamente, siendo una de sus causas la actividad no planificada del hombre, es decir, el desarrollo social, económico y tecnología conlleva un costo para el medio ambiente el cual el ser humano ha ignorado o no ha sabido ponderar, lo que lo lleva a realizar acciones no autosustentables.

Ahora bien Se identifican como conflictos ambientales las controversias de información, intereses o valores entre al menos dos grupos independientes, referidas a cuestiones relacionadas con el acceso, disponibilidad y calidad de los recursos naturales y de las condiciones ambientales del entorno que afectan la calidad de vida de las personas. (Rosario, 2008)

Es decir, un conflicto ambiental involucra a extractos de la sociedad determinados, tales como colonos, pueblos indígenas, comunidades, organizaciones locales, que se ven afectados de manera directa o indirecta por la negligencia y falta de planeación de las instituciones, sobre todo aquellas encargadas de otorgar autorizaciones para el desarrollo de indistintos proyectos, estos conflictos ambientales surgen como contraposición entre aquellos que pretenden contaminar el medio ambiente, o bien que autorizan a otra personas a realizar dicha contaminación, y aquellos que sufren las consecuencias de la contaminación. (Choren)

Mientras que un conflicto socioambiental es una afectación directa a grandes grupos sociales a través del deterioro del medio ambiente, en donde somos todos los habitantes quienes sentimos las repercusiones del daño reiterado al mismo.

Sin embargo los diversos conflictos con los cuales cuenta nuestro medio ambiente, cuentan de igual forma con diversos génesis, siendo uno de ellos, los nuevos valores post-materialistas, los cuales surgen en los países desarrollados al ver sus necesidades fisiológicas cubiertas, es decir, techo, alimentación vestido, situación que les permite enfocarse en cuestiones no materiales, tales como el medio ambiente, los valores y los derechos humanos, teniendo como consecuencia el surgimiento de partidos verdes, y movimientos sociales pacifistas, con intereses ajenos a lo material, que se caracterizan por el pluralismo de valores y la participación en las decisiones del interés colectivo. (Walter, 2009) 
Otro de los génesis de los conflictos ambientales son los denominados conflictos ecológicos distributivos, en donde son las asimetrías o desigualdades sociales, geográficas y temporales del uso de los recursos naturales que hace el hombre, lo que genera el conflicto entre las poblaciones, es decir, el uso desmedido que hacen algunos de los recursos naturales por un lado, y la afectación de aquellos que subsisten directamente en convivencia con el medio ambiente. (Martínez Alier, 1997)

Debemos comprender que los conflictos ambientales no son solo disputas o desacuerdos por la propiedad y/o el uso de un recurso, sino la dicotomía entre ambiente y vida, es decir, por un lado el medio ambiente y sus recursos es considerado como espacio económico y de comercio, y por otro lado, como el espacio vital donde surge y se desarrolla la vida. (Sabatini, 1997)

Es por eso que podemos determinar que los conflictos ambientales son de igual forma, un tema cultural, en donde debemos preocuparnos por generar conciencia social-ambiental, desde temprana edad, a efecto de formar sujetos con conciencia ambiental e interés social, y no solo personas con intereses personales, es decir, si no logramos un cambio paradigmático en la cultura de la sociedad en el tema ambiental, no podremos hablar de una solución de fondo a los conflictos ambientales que nos aquejan el día de hoy y los cuales se están viendo acrecentados.

Es aquí donde tiene cabida la mediación en materia ambiental, como mecanismos culturizados y pacificador, dotando a las personas de nuevas herramientas de convivencia y dialogo.

\section{LA MEDIACIÓN COMO RESPUESTA DE FONDO A LOS CONFLICTOS AMBIENTALES}

A efecto de poder determinar la mejor opción para la resolución de conflictos en materia ambiental, con miras a la no reincidencia de los mismos, es menester señalar la clasificación de estas conflictivas, la cual tomamos de la realizada por Christopher Moore, quien las plasma de la siguiente manera.

Por principio de cuenta a) por información, cuando la disputa se suscita por desacuerdos en las fuentes, análisis o interpretación de la información, b) por relaciones, cuando el génisis del conflicto es la falta de confianza, de credibilidad o la duda sobre las partes, c) por intereses, al ser el objeto del conflicto, posiciones, necesidades, temores y preocupaciones, d) estructurales, cuando las partes encuentras limites físico, estructurales, institucionales o bien formales, los cuales les impiden resolver su conflictiva y por último e) por valores, al disputarse tablas y 
conceptos de valores entre las partes, siendo este tipo de conflictos los más complicados de resolver, toda vez que ir en contra del valor e idiosincrasia de una persona es ir en contra de la persona misma, en donde no podemos esperar un cambio radical en su constitución personal, solo hacerle ver a la contraparte que existen distintas percepciones en torno al conflicto, las cuales también pueden ser válidas. (Moore, 1989)

En nuestro país existe una extensa cultura legal de protección al medio ambiente, tanto a nivel nacional como a nivel local, las cuales han sido reforzada por tratados internacionales, en específico por el instrumento jurídico suscrito entre nuestro país con los Estados unidos de América y Canadá, entrando en vigor en 1994 y que al mismo tiempo originó la creación de la Comisión para la Cooperación Ambiental. (Figueroa Diaz, 2008)

Situación por la cual se ha vuelto una obligación por parte del Estado y de la ciudadanía como coparticipes y coadyuvantes, para la protección, promoción y salvaguarda de los recursos naturales y el medio ambiente en genera, contando con diversos sistemas e instrumentos legales para ello, de los que destaca la Ley General del Equilibrio Ecológico y la Protección Ambiental y la Ley Federal de Responsabilidad Ambiental.

Señalando la Ley General del Equilibrio Ecológico y la Protección Ambiental que en los casos en los que una denuncia popular no vulnere la normatividad ambiental, o afecte cuestiones de orden público e interés social, dicha denuncia podrá sujetarse para su resolución a un proceso de conciliación, además de estipular de manera expresa como forma de terminación de una denuncia popular, el mecanismo de conciliación, que si bien es cierto no habla de manera directa de la mediación, no menos cierto es que la mediación es la columna vertebral de la conciliación. ${ }^{1}$

De igual forma la Ley de Responsabilidad Ambiental, contempla en su título segundo, capitulo primero, la utilización de los mecanismos alternos de solución de conflictos, entre ellos la mediación, para resolver los conflictos de carácter ambiental, hasta antes de que se dite sentencia definitiva y en asuntos que de generar responsabilidad penal, sean delitos susceptibles de permitir el perdón del ofendido, así como el desinterés jurídico de la víctima y de la procuraduría. ${ }^{2}$

Situación con la cual diferimos, toda vez que se debe de permitir el uso de la mediación en todas las etapas del proceso, ya sea pre-procesal, intra-procesal o bien post-procesal, esto atendiendo a la dualidad de la mediación, es decir, el conceptualizar a la mediación no solo como un método

\footnotetext{
${ }^{1}$ V. Artículo 196 y 199 de la Ley General del Equilibrio Ecológico y la Protección al Ambiente

${ }^{2}$ V. Artículos 47 a 51 de la Ley Federal de Responsabilidad Ambiental
} 
de resolución de conflictos, el cual es más económico y veloz, sino también como un mecanismo reparador de relaciones interpersonales, que dota de nuevas herramientas de socialización a las partes, basadas en el dialogo y en la empatía, lo cual a la postre logrará la reducción del índice de reincidencia en este tipo de conflictos.

Es decir, se debe de permitir el uso de la mediación en todas las etapas del proceso, aun cuando su finalidad no sea la de deslindar y/o atribuir responsabilidades a las partes, aunado a lo anterior, debemos señalar que consideramos erróneo el limitar el uso de la mediación para asuntos que al generar responsabilidad penal, se trate de delitos que permitan el perdón del ofendido o bien el desinterés jurídico de la víctima, toda vez que no se está tomando en cuenta las bondades pacificadoras de la mediación, así como su capacidad de reparar relaciones interpersonales, y se le está encajonando como un mecanismo de pronta respuesta, que solo funciona para reducir exceso de trabajo de los tribunales de justicia formal.

Por lo antes analizado, surge la imperiosa necesidad de resolver los conflictos ambientales con miras hacia su no repetición, tal como es el objetivo de los diversos sistemas de resolución de conflictos, ya sea que se basen en la autocomposición, como la mediación o bien en la heterocomposición

Sin embargo la utilización de la mediación en materia ambiental no ha convencido a todos, y cuenta con detractores, señalando diversas fallas en el mecanismo, las cuales lo hacen inviable para ser utilizado en una materia de repercusión global, algunos de los puntos negativos que se le realizan, es la complicación que puede provocar al intentar determinar con exactitud los afectados en este tipo de conflictos, sin embargo esto sucede puesto que existe una confusión en relación a los conceptos de conflicto ambiental con problema ambiental, diferenciación que se realizó con anterioridad, y en donde se señaló que en los conflictos ambientales es posible determinar de manera precisa el o los afectados directos por las acciones u omisiones que generen a la postre un problema ambiental, así como es también posible determinar la institución u organización que lleva acabo la acción lesiva o bien que otorga el permiso a otra, para que la realice, lo anterior a efecto de determinar el interés legítimo o jurídico de las partes.

En este sentido la doctrina ha acuñado un término para denominar a las personas involucradas en un conflicto ambiental, utilizando el término stakeholders, este término es más amplio que la simple concepción que conocemos sobre las partes de un conflicto y a la cual estamos tan acostumbrados, este concepto es tan amplio como los términos manejados por la justicia 
restaurativa, al contemplar a todas las personas que se pudieren ver afectados con el conflicto y con la posible solución de éste, entre estos encontramos al Estado, al sector empresarial y al sector ciudadano, por lo general, en materia ambiental, las partes suelen ser un cumulo de sujetos y no personas en lo individual. (Ruiz, 2011)

\section{CONCLUSIONES}

Por lo antes señalado, consideramos que la mediación ambiental es el complemento necesario que viene a dotar de verdadera funcionabilidad al derecho ambiental a nivel nacional e internacional, toda vez que se ha convertido en el complemento perfecto, al generar opciones menos lesivas para las partes, así como acuerdos que son tomados realmente tomados en cuenta por los mismos, toda vez que ellos han sido sus artífices, además de implementar su política de prevención utilizando la vergüenza y no la sanción como mecanismo de modificación de conducta, tal como lo establece la teoría de la vergüenza reintegrativa, y finalmente, nos ayuda a delimitar de manera clara y precisa los problemas netamente ambientales, diferenciándolos de los problemas comerciales o industriales, evitando de esta manera el conflictuar por cuestiones secundarias que no conforman el génesis o la esencia del conflicto y que por consecuencia no podríamos hablar de una resolución profunda y total del mismo, lo cual nos llevaría al desgaste de las partes, un desgaste emocional, así como en sus relaciones personales, un desgaste económico sin mencionar una pérdida de tiempo al no ser capaces de resolver la conflictiva de raíz. (Figueroa Diaz, 2008)

Son estas algunas de las cuestiones por las cuales consideramos que la mediación en materia ambiental no solo debe de ser permitida como opción, sino que debe de convertirse en un pilar inamovible en la resolución de conflictos ambientales, por las diversas bondades que acarrea y el impacto que potencialmente son capaces de producir estos conflictos. 


\section{Bibliografía}

Ávila Verdín, E. G., Rosas- Topete, N., \& Arciniega-Ponce, A. (2014). La cultura del agua desde la percepción ciudadana. Revista Iberoamericana de Producción Académica y Gestión Educativa, 1-20.

Bazemore, G., \& Walgrave, L. (1999). Restorative Juvenile Justice. Missouri: Willow Tree.

Caballero Sepulveda, E. (2009). Principios básicos sobre la utilización de programas de justicia restaurativa en materia penal adoptados por las Naciones Unidas.

Cervantes Bravo, I. G. (2012). La justicia restaurativa como elemento fundamental en el sistema acusatorio de Nayarit. En S. A. Moran Navarro, I. G. Cervantes Bravo, \& H. Lomeli Payan, El sistema acusatorio oral de Nayarit a debate (págs. 51-64). Tepic, Nayarit, México: fontamara.

Figueroa Diaz, L. (2008). Reflexiones en torno a la mediación y la efiacia del derecho ambiental. Alegatos, 143-156.

Hidalgo Murillo, J. D. (2010). Justicia altenrativa en el proceso penal mexicano. México: Porrúa.

Kemelmajer, A. (s.f.). En busca de la tercera vía, la llamada justicia restaurativa reparativa, reintegrativa o restitutiva.

Márquez Algara, M. G. (2004). Mediacion y adminsitracion de justicia, hacia la consolidacion de una justicia participativa. México: Universidad Autonoma de Aguascalientes.

Marshall, T. (1999). Restorative Justicie: An overview. Gan Bretaña: Home Office.

Martínez Alier, J. (1997). Conflictos de Distribución Ecológica. Andina. 
Moore, C. (1989). Utilizing Negotiations to Resolve Complex Environmental Dispute. En W. Viessman, \& E. Smedron, Managing Water-Related Conflicts: The Engineer`s Role. New York.

Pachecho Pulido, G. (2012). Mediacion, cultura de la paz, medio alternativo de adminsitracion de justicia. México: Porrua.

Pastrana Aguirre, L. a. (2009). La mediación en el sistema procesal acusatorio en México, doctrina y disposiciones legales. México: Flores.

Ruiz, A. (2011). Chile.

Sabatini, F. (1997). Conflictos ambientales en América Latina: ¿distribución de externalidades o. Estudios Sociales.

Soleto Muñoz, H., \& Ortero Parga, M. (2007). Mediacion y solucion de conflictos, habilidades para una necesidad emergente. España: Tecnos.

Vannes, D. W., \& Strong, K. H. (2001). Restoring Justice. Argentina: Rústica.

Zaera Navarrete, J. I. (2013). Guia practica de meddiacion, 10 preguntas y respuestas para abogados. Valencia: Tirant Lo Blanch.

Walter, M. (2009). Conflictos ambientales, socioambientales, ecológico distributivos, de contenido ambiental...Reflexionando sobre enfoques y definiciones. Centro de investigación por la paz, 2-9.

Choren, S. (s.f.). www.cricyt.edu. Recuperado el 03 de Febrero de 2016, de http://www.cricyt.edu.ar/enciclopedia/terminos/ConflAamb.htm 
Rosario, U. d. (2008). www.urosario.edu. Recuperado el 03 de Febrero de 2016, de http://www.urosario.edu.com/Univerisdad-Ciencia-Desarrollo/ur/FasciuculosAnteriores/Tomo-III-2008/Fasciculo-12/ur/Que-es-un-conflicto-ambiental/ 